\title{
Adoption of an Electronic Medical Record Tool for Childhood Obesity by Primary Care Providers
}

\author{
Amy Williams ${ }^{1} \quad$ Christy Turer $^{2} \quad$ Jamie Smith $^{1} \quad$ Isabelle Nievera $^{3} \quad$ Laura McCulloch $^{4}$ Nuha Wareg ${ }^{1}$ \\ Megan Clary ${ }^{5}$ Anuradha Rajagopalan ${ }^{5}$ Ross C. Brownson ${ }^{6,7}$ Richelle J. Koopman ${ }^{1}$ Sarah Hampl $\left.\right|^{8,9}$
}

\footnotetext{
${ }^{1}$ Department of Family and Community Medicine, University of Missouri, Columbia, Missouri, United States

2 Department of Internal Medicine-Pediatrics, University of Texas Southwestern, Dallas, Texas, United States

3 University of Missouri School of Medicine, Columbia, Missouri, United States

${ }^{4}$ Columbia/Boone County Public Health and Human Services, Columbia, Missouri, United States

${ }^{5}$ Department of Child Health, University of Missouri, Columbia, Missouri, United States

${ }^{6}$ Department of Surgery and Alvin J. Siteman Cancer Center,

Washington University in St. Louis, St. Louis, Missouri, United States

${ }^{7}$ Division of Public Health Sciences, Department of Surgery, Alvin J. Siteman Cancer Center, Washington University School of Medicine, Washington University in St. Louis, St. Louis, Missouri, United States

${ }^{8}$ General Pediatrics and Weight Management, Children's Mercy Hospital Center for Children's Healthy Lifestyles and Nutrition, Kansas City, Missouri, United States

${ }^{9}$ Department of Pediatrics, University of MO-Kansas City School of Medicine, Kansas City, Missouri, United States
}

Appl Clin Inform 2020;11:210-217.
Address for correspondence Amy Williams, MD, MSPH, Department of Family and Community Medicine, University of Missouri, MA306 Medical Sciences Building, 1 Hospital Drive, Columbia, MO 65212, United States (e-mail: williamsamy@health.missouri.edu).

\section{Abstract}

Keywords

- electronic health records and systems

- infants and children

- ambulatory care/primary care

- adoption

- implementation and deployment
Background Primary care providers are tasked with the increasingly difficult job of addressing childhood obesity during clinic visits. Electronic medical record (EMR)enabled decision-support tools may aid providers in this task; however, information is needed regarding whether providers perceive such tools to be useful for addressing nutrition and physical activity lifestyle behaviors.

Objectives This study aimed to evaluate the usefulness and usability of FitTastic, an EMR-enabled tool to support prevention and management of childhood obesity in primary care.

Methods In this mixed-method study, we implemented the FitTastic tool in two primary-care clinics, then surveyed and conducted focused interviews with providers. Validated Technology Acceptance Model perceived usefulness and National Aeronautics and Space Administration (NASA) perceived usability survey questions were emailed to 60 providers. In-depth provider interviews with family medicine and pediatric physicians $(n=12)$ were used to further probe adoption of FitTastic.

Results Surveys were completed by $73 \%$ of providers $(n=44)$. The mean score for FitTastic's usefulness was 3.3 (standard deviation [SD] $=0.54$, scale $1-5$, where 5 is strongly agree) and usability, 4.8 (SD $=0.86$, scale $1-7$, where 7 is strongly agree). Usefulness and usability scores were associated with intention to use FitTastic received

December 3, 2019

accepted

January 23, 2020 (c) 2020 Georg Thieme Verlag KG Stuttgart · New York
DOI https://doi.org/ 10.1055/s-0040-1705106. ISSN 1869-0327. 
(correlation for both, $p<0.05$ ). Data from provider interviews indicated that useful features of FitTastic included: standardizing the approach to childhood obesity, and facilitating conversations about weight management, without increasing cognitive workload. However, use of FitTastic required more time from nurses to input lifestyle data.

Conclusion FitTastic is perceived as a useful and usable EMR-based lifestyle behavior tool that standardizes, facilitates, and streamlines healthy lifestyle conversations with families. Perceived usability and usefulness scores correlated with provider intentionto-use the technology. These data suggest that EMR-based child obesity prevention and management tools can be feasible to use in the clinic setting, with potential for scalability. Usefulness can be optimized by limiting amount of time needed by staff to input data.

\section{Background and Significance}

Childhood obesity is a public health epidemic that tracks into adulthood with significant health consequences. Primary care providers are tasked with screening and managing childhood obesity during time-constrained clinic visits that have competing tasks, ${ }^{1}$ in an increasingly complex work environment. ${ }^{2}$ Although data indicate that providers recognize that childhood obesity is an important medical issue, providers cite low proficiency in childhood-obesity prevention and treatment counseling as a key barrier to address childhood obesity. ${ }^{3,4}$ The electronic medical record (EMR) offers a novel way to support providers in delivering childhood-obesity prevention and management counseling ${ }^{5,6}$ and to complement emerging technology 7,8 to detect children who are at risk for childhood obesity. EMR-based solutions, however, are most successful when they are perceived by providers as useful and easy to use. ${ }^{9}$ Data from a study assessing predictors of adoption of health information technology indicate that perceived ease of use is associated with provider intention to use the technology. ${ }^{9}$

According to the Technology Acceptance Model (TAM) theory, a widely recognized model of technology-behavior intention, ${ }^{10}$ use of technology is influenced by user attitude, perceived usefulness, and perceived ease of use. ${ }^{10}$ The focus of the most updated version of the TAM and TAM2 is on intent to use and actual use of technology (-Fig. 1). ${ }^{9}$

Another key facilitator of technology acceptance is the ability to reduce cognitive workload, defined as the mental effort required by an individual to complete a task. ${ }^{11}$ The

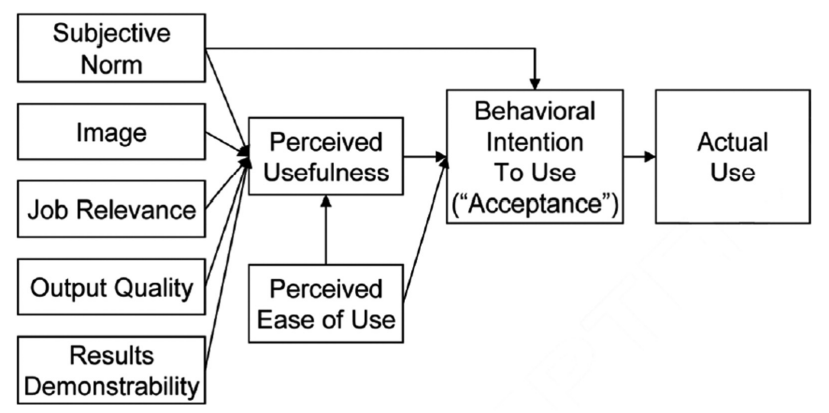

Fig. 1 Technology acceptance model (TAM) $2 .^{9}$
National Aeronautics and Space Administration-Task Load Index (NASA-TLX) is a widely used method to determine the impact of a technology, including EMR-based decision-support tools, on a user's cognitive workload. ${ }^{12}$ NASA-TLX items measure the efficiency of technologies as they restructure tasks. ${ }^{13}$ For example, the ideal EMR tool to support providers in addressing childhood obesity would minimally restructure the tasks involved at each clinic visit, while lowering the amount of mental calculations needed to complete each task. NASA-TLX helps to understand the extent to which their tool gives concise instructions, minimizes unnecessary requests for information, limits the number of different screens needed to complete tasks, and requires the least number of mouse clicks possible. ${ }^{14}$ NASA-TLX-item scores indicate how a tool might be improved further to lower a provider's cognitive workload, ease use, and maximize efficient and effective execution of complex tasks. ${ }^{13}$

"12345-FitTastic," hereafter referred to as FitTastic (www. fit-tastic.org), is a community-designed EMR tool designed to support primary-care providers in addressing the following five recommended nutrition and physical activity behaviors aiding childhood obesity prevention ${ }^{15,16}$ : (1) at least 1 hour of physical activity a day, (2) 2 hours or less of screen time a day, (3) three servings of low-fat milk or calcium a day, (4) at least four servings of water a day instead of sugary drinks, and (5) at least five servings of fruits and vegetables a day. The FitTastic healthy lifestyle assessment evaluates a child's habits in each of these five areas, and can be completed by a nurse or other clinic staff. Providers utilize the healthy lifestyle assessment when communicating with families about selecting a lifestyle goal. In a 2018 cross-sectional study of 24,255 patients, adherence to a greater number of FitTastic behaviors was inversely associated with obesity in a dose-dependent manner. ${ }^{17}$

Although there is an identified need for EMR-based tools to assist primary care providers in preventing and managing childhood obesity, there is little information about whether these tools are perceived by providers as useful and usable and if they would be accepted by providers if implemented in primary care clinics. Therefore, we implemented the FitTastic tool in two clinics. Then, guided by the TAM2 model ( - Fig. 1), we surveyed and interviewed providers to determine provider 
perceptions regarding the usefulness and usability of the FitTastic tool.

\section{Objectives}

We aimed to determine provider perspectives regarding the usefulness and usability of the EMR-based FitTastic lifestyle behavior tool and to understand other factors related to provider adoption of EMR-based clinic support tools.

\section{Methods}

At least 3 months following training and implementation of FitTastic (implemented between August 2016 and February 2017), we performed a mixed-method study. Provider surveys (conducted during August-October 2017) were used to determine rates of the reported use, usefulness, and usability of FitTastic, and impact of using FitTastic on cognitive workload. Then, focused interviews (conducted at April-June 2017) with family medicine and pediatric primary-care providers were used to further probe the usefulness and usability of FitTastic. Provider surveys were completed with verbal informed consent and an institutional review board (IRB)-approved waiver of written consent. Focused interviews were conducted after obtaining written informed consent from providers.

FitTastic was implemented at two suburban ambulatory clinics staffed by 60 providers, 28 pediatricians ( 10 attendings and 18 residents) and 32 family-medicine physicians (22 attendings and 10 residents). Implementation included use of prepared scripts and documentation guides to conduct trainings with staff, nurses, and providers at four consecutive monthly clinic meetings, then quarterly, and at resident didactic conferences (held biannually). FitTastic was first implemented at the pediatric clinic (starting in August 2016), then 6 months later at the family-medicine clinic (February 2017).

Surveys regarding FitTastic lifestyle behaviors were completed by families of all children 3 to 17 years old when presenting for well-child visits. Nurses adjudicate child maturity and health literacy to support families in completing all well-child visit-related forms (not solely the behavior assessment) independently versus via interview, then enters responses into the EMR (see - Fig. 2 for screen shot of data entry). The provider uses the information to identify modifiable behaviors and encourage them to set goals to change them. The data are immediately available when the nurse enters it into the EMR; there is no lag. There is an autotext function that pulls the data into the note if precharted. During the visit, health care providers worked with families to develop a healthy lifestyle goal for one or two behaviors. Providers or nurses gave families an educational handout and an incentive matched to one agreed upon lifestyle goal (for example, a recreation-center pass to a family who selected to increase physical activity, or a grocery store voucher to purchase fruits/vegetables to a family who chose to increase fruits and vegetables). Families were unaware of available incentives or that an incentive was going to be provided prior to completing their FitTastic assessment or selecting their goals. Surveys regarding FitTastic use, usefulness, and usability were e-mailed to providers 6 to 12 months after FitTastic implementation.

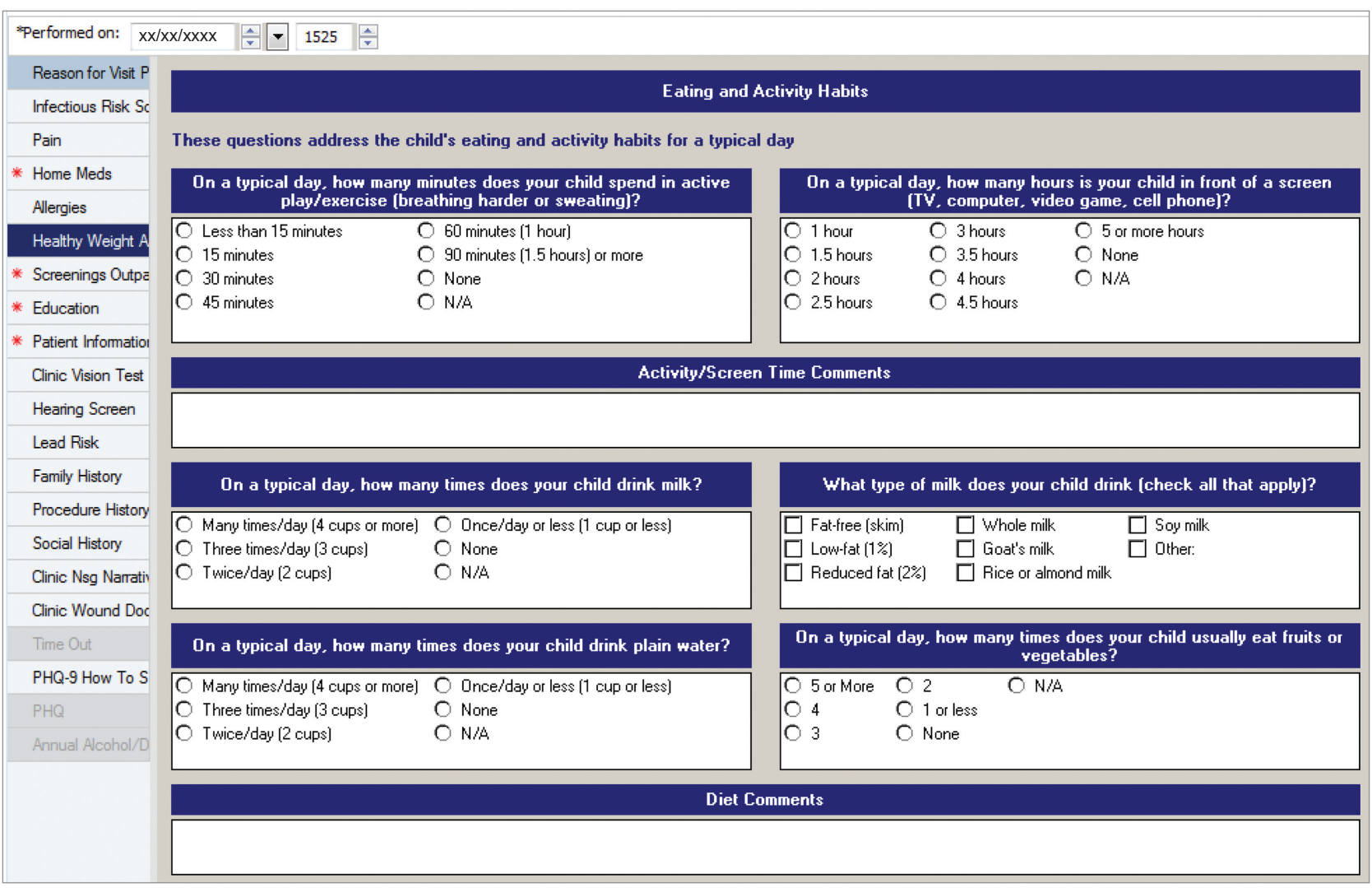

Fig. 2 FitTastic lifestyle assessment. 


\section{Quantitative Analysis of Usefulness and Usability}

Usefulness of FitTastic was determined using a 15-item Perceived Usefulness Scale that asked providers to rate (Likert's scale of 1-5) the extent to which, when communicating about lifestyle behaviors with all families, use of FitTastic saved time, enhanced effectiveness, improved performance, and improved the ease of communicating about a child's weight to the parent and child. Scores for individual survey items were averaged, and a score of 2.5 (on a scale of 1-5) interpreted as neutral (e.g., the provider neither agreed nor disagreed that FitTastic was useful for the specific item). The Perceived Usefulness Scale has a high reliability $(\alpha=0.97) .^{18}$

Usability of FitTastic was determined using the 19-item NASA Perceived Usability Scale. NASA-TLX items ask providers to rate (on a Likert's scale of 1-7) the extent to which FitTastic was "simple to use," "provided easy-to-understand info," allowed workers to "complete work effectively, quickly, and efficiently," and "had a pleasant interface." We used the NASATLX Usability Scale because of its focus on understanding how a technology impacts a user's workload in a cognitively complex work environment, ${ }^{19}$ good reliability (Cronbach's $\alpha$ coefficient and split-half reliability both $>0.80$ ), and structure validity (based on factor analysis ${ }^{20}$ ). Scores for individual items were averaged, and a score of 3.5 (scale of 1-7) was interpreted as neutral.

Descriptive statistics were used to describe providerreported impact of FitTastic on rates of education and management of childhood obesity, time to administer FitTastic, time using FitTastic to communicate with families during visits, and desire to continue use of FitTastic at well-child visits (and how the absence of prizes might affect desire to continue FitTastic use). Differences in responses by provider specialty (e.g., family practice or pediatrics) and role (e.g., attending physician or resident/trainee) were tested using the Chi-square test of binomial proportions. Perceived usefulness and usability of FitTastic were examined using item scores.

\section{Qualitative Methodology}

Questions for the provider interviews were developed to probe the practical experiences of providers when addressing lifestyle behaviors with all children (including obesity prevention in healthy weight children) using FitTastic in primary care clinics. Questions were compiled into a moderator's guide with oversight by a trained qualitative researcher (R.K.) and clinical researchers with experience addressing child obesity and implementing FitTastic in their primary care clinics (A.W. and S.H.). To assess physician perception of FitTastic among clinicians who heavily used versus rarely used the tool (determined using EMR analytic software), we stratified focused interviews by high provider use of FitTastic (FitTastic used at $>80 \%$ of well child visits, $n=4$ ), moderate use (FitTastic used at $20-80 \%$ of well child visits, $n=4$ ), and low use (FitTastic used at $<20 \%$ of well child visits, $n=4)$. According to standard practice in the literature, $^{21}$ we concluded obtaining further interviews upon achieving thematic saturation, that is, thematic coding of each additional interview revealed duplication of existing codes/themes with no new themes emerging.
Two trained research assistants (N.W. and L.M.) conducted open-ended semistructured interviews with a purposeful sample of six family medicine and six pediatric physicians. All providers approached agreed to be interviewed. First, a trained research assistant described the study aims, answered questions, and obtained written informed consent to audio record the interview. Then, the research assistant asked providers open-ended questions about their (1) approach to screening for and managing childhood obesity, (2) general barriers in addressing childhood obesity, and (3) specific barriers to implementation of FitTastic. Interviews (conducted between April and June 2017) lasted 30 to 60 minutes and providers received a $\$ 40$ participation incentive.

Audio recorded interviews were transcribed verbatim by a professional transcription service. Transcripts were analyzed using Dedoose qualitative software. Then, two reviewers (A.W. and N.W.) independently used the results to conduct thematic analysis. Coding discrepancies were resolved by consensus or discussion with the research team. Duplication of codes and themes suggested sufficient data saturation. We discussed and compared themes from the interviews that pointed to specific TAM2 perceived usefulness items, usability factors, and barriers and facilitators to FitTastic use. Themes were used to understand barriers to and facilitators of FitTastic use, usefulness, and usability.

\section{Results}

Of 60 providers who implemented FitTastic, 44 (73\%) completed the survey. Participants identified as the following: 40\% male and 60\% female; 77\% White, 13\% Asian, 4\% African American, 2\% Hispanic, and 4\% did not disclose their race or ethnicity. Five providers reported they did not conduct wellchild visits and were removed. The final survey sample included 39 providers. Of these, 59\% were attendings and $41 \%$ were residents and $67 \%$ were family-medicine trained and $33 \%$ were pediatricians

\section{Usefulness}

Nearly two in three (64\%) providers reported that FitTastic increased their rates of both childhood obesity screening and education, whereas $18 \%$ reported FitTastic had no impact on either. For time spent talking with patients using FitTastic, $33 \%$ of providers reported talking less than 2 minutes, $48 \%$ reported 2 to 3 minutes, and $12 \%$ reported 4 to 5 minutes. Ninety percent of providers stated that they would continue use of FitTastic in their clinics, including 69\% who would continue using FitTastic even without patient incentives. Responses did not differ by provider type (family medicine versus pediatrics) nor by provider role (attending versus residents; $p>0.05$ ).

The mean score of FitTastic on the Perceived Usefulness Scale was a $3.3(\mathrm{SD}=0.5, \alpha=0.9)$ on all 15 items on the 1 to 5 Likert's scale, ( - Table 1). The mean score for the 19 items on the NASA-TLX Usability Scale was a $4.8(\mathrm{SD}=0.9, \alpha=0.95)$ on the 1-7 Likert scale, ( - Table 1). Usefulness and usability scores did not differ by provider type or provider role. 
Table 1 Perceived usefulness and NASA usability scores of FitTastic

\begin{tabular}{|c|c|c|c|c|}
\hline & $\mathrm{N}$ & Mean & SD & $\alpha$ \\
\hline \multicolumn{5}{|l|}{ TAM } \\
\hline Perceived usefulness mean score & 38 & 3.3 & 0.5 & .91 \\
\hline \multicolumn{5}{|l|}{ Perceived usefulness items } \\
\hline $\begin{array}{l}\text { If FitTastic were available to other providers, colleagues, and clinics, } \\
\text { how likely would you be to recommend use of it? }\end{array}$ & & 3.9 & 0.6 & \\
\hline Overall, I find FitTastic useful in my job & & 3.7 & 0.6 & \\
\hline Using FitTastic improves the quality of the work I do & & 3.6 & 0.7 & \\
\hline Using FitTastic enhances my effectiveness on the job & & 3.6 & 0.7 & \\
\hline FitTastic supports critical aspects of my job & & 3.6 & 0.7 & \\
\hline Using FitTastic makes it easier to do my job & & 3.5 & 0.7 & \\
\hline Using FitTastic improves my job performance & & 3.5 & 0.7 & \\
\hline FitTastic addresses my job-related needs & & 3.4 & 0.9 & \\
\hline FitTastic enables me to accomplish tasks more quickly & & 3.2 & 1.0 & \\
\hline Using FitTastic saves me time & & 3.1 & 1.0 & \\
\hline Using FitTastic allows me to accomplish more work & & 3.1 & 0.8 & \\
\hline Using FitTastic gives me great control over my work & & 3.0 & 0.8 & \\
\hline Using FitTastic increases my productivity & & 3.0 & 0.9 & \\
\hline Using FitTastic reduces the time I spend on unproductive activities & & 3.0 & 0.9 & \\
\hline Managing child obesity would be difficult to do without FitTastic & & 2.6 & 0.8 & \\
\hline \multicolumn{5}{|l|}{ NASA } \\
\hline Usability mean score & 37 & 4.8 & 0.9 & .95 \\
\hline \multicolumn{5}{|l|}{ Usability items } \\
\hline FitTastic simple to use & & 5.5 & 1.1 & \\
\hline Easy to learn FitTastic & & 5.2 & 1.3 & \\
\hline FitTastic provides clear information & & 5.2 & 1.0 & \\
\hline FitTastic provides easy-to-understand information & & 5.2 & 1.1 & \\
\hline Info provided effective for task completion & & 5.2 & 1.1 & \\
\hline Complete work effectively using FitTastic & & 5.1 & 1.1 & \\
\hline Satisfaction with ease of FitTastic use & & 5.1 & 1.3 & \\
\hline Complete work efficiently using FitTastic & & 5.1 & 1.2 & \\
\hline Complete work quickly using FitTastic & & 5.0 & 1.2 & \\
\hline Clear info organization on FitTastic screens & & 5.0 & 1.2 & \\
\hline Comfortable using FitTastic & & 5.0 & 1.4 & \\
\hline Easy to find information needed & & 4.9 & 1.1 & \\
\hline Overall satisfied with system & & 4.8 & 1.1 & \\
\hline Became productive quickly using FitTastic & & 4.7 & 1.3 & \\
\hline System interface is pleasant & & 4.4 & 1.2 & \\
\hline System has all functions and capabilities & & 4.4 & 1.2 & \\
\hline I like using interface & & 4.3 & 1.2 & \\
\hline Error recovery quick and easy & & 3.9 & 0.8 & \\
\hline FitTastic error messages tell how to fix problems & & 3.9 & 0.9 & \\
\hline
\end{tabular}

Abbreviations: NASA, National Aeronautics and Space Administration; SD, standard deviation; TAM, technology acceptance model. Note: TAM perceived usefulness scale range is 1 to 5 , neutral is 2.5 . The NASA usability scale range is 1 to 7 , neutral is 3.5 . 


\section{Qualitative Results}

Of 12 providers interviewed, 50\% were female, 75\% were Caucasian $17 \%$ were Asian, and $8 \%$ were African American. All of the interviewed providers were supervising/attending physicians. Usefulness themes that emerged from the interviews corresponded well with two Perceived Usefulness domains of the TAM2 model were "job relevance" and "output quality" (-Table 2 ).

The theme "job relevance" was used to convey that providers noted that FitTastic appropriately supports their need to address childhood obesity as part of their job. For example, one provider said "FitTastic is a great screening tool. [After implementing FitTastic] we definitely do a good job about counseling about activity." The theme "output quality" was chosen to encapsulate providers reporting that FitTastic supports their effectiveness in counseling about childhood obesity. One provider stated "I was always pleasantly surprised at how interested the children were and how excited they were about what they'd selected."

Additional themes regarding facilitators and barriers of FitTastic's usability and impact on time, visit structure, cognitive load, and application to patients are shown in -Table 2 . The biggest barrier that we identified was that FitTastic slowed down the workflow and increased clinic visit time for some providers. One provider stated, “... it's slowed the nurse down, acquiring the information. You know, 20-minute visits and it takes 10 minutes to get a patient in a room so that tends to get us quite behind." The other usability themes were facilitators for FitTastic's use. Providers liked the structure that FitTastic added to their conversations with families regarding healthy lifestyles. One provider said "I think that it has provided a lot of structure to something that had been more free form in the past and makes it easier to get it done." Providers also liked that FitTastic did not increase their clinical cognitive workload. One provider stated, "it's simple, it's something we can remember." Finally, the last usability theme was that FitTastic reduced patient defensiveness to discuss lifestyle changes increasing family acceptance, as one provider noted "I think when I described my approach before we would start at the body mass index (BMI) percentiles, assessing that the child didn't have a healthy weight, and then saying "well, let's talk about some of their habits," and that maybe felt a little bit more defensive."

\section{Discussion}

Study data suggest that, in general, providers report that the FitTastic lifestyle behavior EMR-based decision support tool improves their rates of childhood obesity prevention and management, is useful and usable, supports the task of counseling about childhood obesity, and increases the quality of this counseling. These scores correlate well with provider intention to continue using the tool, consistent with the TAM2 model.

Thematic analysis of interview transcripts identified that the adoption of the EMR-based clinical support tool was facilitated by its structured approach to lifestyle behavior assessment, neutral cognitive workload, and positive patient application. A barrier that was identified, however, was the amount of time required for nurses to enter data into the EMR. Although most providers reported spending 3 minutes or less using the tool, additional time was needed by front desk staff and nurses to administer the survey, enter survey data, and explain the program. Thus, although FitTastic does not appear to slow down well-child visits, providers reported in the interviews that the time required for nurses to conduct the healthy behavior screening was disruptive. These results are consistent with a previous study which identified time constraints as a significant barrier in addressing childhood obesity. ${ }^{1}$ A key facilitator of adoption of an EMR system is speed. ${ }^{10}$ Busy practitioners will not readily adopt a technology that is perceived to slow down clinic visits. ${ }^{22}$

Quantitative and qualitative results suggest that the FitTastic tool reduced, or at least did not significantly add to cognitive workload. Reducing cognitive workload is important to prevent increased information chaos which can increase physician burnout and medical errors. ${ }^{2}$ The favorable usability themes aligned well with the mean NASA Usability score of 4.83 (Likert's scale 1-7).

Table 2 Provider interview themes and examples of narratives

\begin{tabular}{|l|l|l|}
\hline Theme & \multicolumn{1}{|l|}{ Explanation } & Example of narrative \\
\hline Perceived usefulness: themes from technology acceptance model-2
\end{tabular}


In addition to the effectiveness and adoption of FitTastic, we assessed its scalability by evaluating its appropriateness and feasibility for expansion into other practices. ${ }^{23}$ An important factor for scalability feasibility is cost. ${ }^{24}$ Start-up effort and cost for EMR-based tools can be significant. A 2017 study of a childhood obesity EMR-based decision support tools for provider and self-guided behavior-change support tools for parents was estimated to cost $\$ 119$ per child or $\$ 237$ per BMI unit reduced. ${ }^{25}$ Implementation of FitTastic in our primary care clinics was facilitated by integration of the software in the EMR before our study. EMR companies can assist the implementation of publicly available tools such as FitTastic by embedding them in their software packages. Goal-matched handouts are available and can be provided online through patient portals in English and Spanish. Cost for goal-matched FitTastic incentives varied from $\$ 1.12$ for each Frisbee to $\$ 3$ for each water tumbler, which can add a substantial and continued expense. After initial grant funding for this project was expended, additional funding to continue providing FitTastic incentives was provided by the Boone County Public Health and Human Services, demonstrating a strong clinic-community public health partnership.

However, although the incentives added a tangible element to the healthy lifestyle conversation and both providers and families enjoyed them, they are not vital. FitTastic originated at Children's Mercy-Kansas City's general pediatrics clinics without incentives, and $69 \%$ of providers in our study indicated they would continue FitTastic even if incentives were not available. We have also piloted the program with goal-matched bracelets and bookmarks, which are less expensive and were also well received. With the exception of routine programming maintenance, costs to maintain an EMR-based childhood obesity tools should be minimal. There are several other healthy lifestyle programs available to assist primary care clinicians address childhood obesity, including the 5-2-1-0 Pediatric Obesity program. ${ }^{26}$ Regardless of which pediatric obesity program a clinic chooses to implement, providers are more likely to adopt it if it is standardized, efficient, and easy to use.

Two major strengths of this study were our high provider survey response rate of $73 \%$ and our use of high, moderate, and low FitTastic-adoption providers for the interviews, which increased the internal validity of the study. Building on the success of this project, we plan to continue evaluating the health impact of this EMR-based tool by examining childhood obesity rates (BMI percentile) and FitTastic behavior rates for the 4 years since it has been implemented. We also plan to evaluate ways to make FitTastic more efficient including patients who have completed the behavior assessment on tablets in the waiting room or before their appointment through patient portals.

\section{Limitations}

There were several limitations to our study. While we were initially able to track provider FitTastic use, after 2 months we experienced technical difficulty, resulting in the loss of this reporting mechanism. The lack of accountability, as well as lack of data for tracking provider progress hindered the implementation of FitTastic. EMR programmers are continuing to work on fixing code for generating the provider reports. Our study was also limited in its use of a sample of family medicine and pediatric providers, which excluded nurses or other clinic staff, who are critical to the implementation success of clinic-based interventions. We will include these key stakeholders in future analysis. Our study was also limited to two clinics at a single Midwest academic health system where the FitTastic was implemented. Although the demographics of the providers interviewed and surveyed are similar to other regional pediatric providers, providers who work at academic institutions, such as the ones included in this study, may be more open to adoption of new technologies such as FitTastic. However, FitTastic has been equally well received at other private practice clinics.

\section{Conclusion}

In this mixed-method study, we found that providers were willing to adopt an EMR-based lifestyle behavior tool, and that perceived usefulness and usability were well-correlated with intention to use the tool. Providers found the tool to be useful to facilitate the conversation to engage families about healthy lifestyle goals, and it did not significantly increase their cognitive workload. Time was not a significant barrier to the use of this tool for most providers, although some reported increased time for nurses to complete and document screenings. The EMR-based lifestyle behavior tool had positive provider adoption and scalability to other primary care clinics. Results from this study can provide helpful insights into the adoption and scalability of other clinicbased EMR innovations.

\section{Clinical Relevance Statement}

Clinical health care providers identify childhood obesity as a significant medical problem but lack EMR-based support to address childhood obesity in primary-care clinics. We found that providers were willing to adopt an EMR-based lifestyle behavior tool in their primary-care practices because it was useful and easy to use. Providers appreciate structured and streamlined approaches to difficult chronic medical problems that ease cognitive workload.

\section{Multiple Choice Questions}

1. In addition to perceived ease of use, which factor of the Technology Acceptance Model 2 (TAM2) predicts behavioral intention to use or accept a new technology?
a. Perceived time to use.
b. Perceived usefulness.
c. Perceived cognitive workload.
d. Perceived cost.

Correct Answer: The correct answer is option b, perceived usefulness. 
2. Providers in this study identified the following as factors that were important to their adoption of the electronic medical record (EMR)-based lifestyle behavior tool, EXCEPT?
a. Application to patients.
b. Structured approach.
c. Cognitive workload.
d. Training difficulty.

Correct Answer: The correct answer is option d, training difficulty.

\section{Protection of Human and Animal Subjects}

This study complies with the World Medical Association Declaration of Helsinki on Ethical Principles for Medical Research Involving Human Subjects and was approved by the 2002856 IRB.

\section{Funding}

This work was supported by the American Academy of Family Physicians Foundation Joint Grant Award Program, grant number: G1603JG. The findings and conclusions in this article are those of the authors and do not necessarily represent the official positions of the American Academy of Family Physicians. Additional support was provided by National Institute of Diabetes and Digestive and Kidney Diseases of the National Institutes of under award numbers 2P30DK092949 and P30DK092950. The findings and conclusions in this article are those of the authors and do not necessarily represent the official positions of the National Institutes of Health.

\section{Conflict of Interest}

None declared.

\section{References}

1 Hill SG, Phan TT, Datto GA, Hossain J, Werk LN, Abatemarco D. Integrating childhood obesity resources into the patient-centered medical home: provider perspectives in the United States. J Child Health Care 2019;23(01):63-78

2 Beasley JW, Wetterneck TB, Temte J, et al. Information chaos in primary care: implications for physician performance and patient safety. J Am Board Fam Med 2011;24(06):745-751

3 Story MT, Neumark-Stzainer DR, Sherwood NE, et al. Management of child and adolescent obesity: attitudes, barriers, skills, and training needs among health care professionals. Pediatrics 2002; 110(1, Pt 2):210-214

4 Barlow SE, Salahuddin M, Butte NF, Hoelscher DM, Pont SJ. Improvement in primary care provider self-efficacy and use of patient-centered counseling to address child overweight and obesity after practice-based changes: Texas childhood obesity research demonstration study. Child Obes 2018;14(08):518-527

5 Morais A, Kelly J, Bost JE, Vaidya SS. Characteristics of correctly identified pediatric obesity and overweight status and management in an academic general pediatric clinic. Clin Pediatr (Phila) 2018;57(10):1168-1175

6 Thaker VV, Lee F, Bottino CJ, et al. Impact of an electronic template on documentation of obesity in a primary care clinic. Clin Pediatr (Phila) 2016;55(12):1152-1159

7 Lingren T, Thaker V, Brady C, et al. Developing an algorithm to detect early childhood obesity in two tertiary pediatric medical centers. Appl Clin Inform 2016;7(03):693-706
8 Dugan TM, Mukhopadhyay S, Carroll A, Downs S. Machine learning techniques for prediction of early childhood obesity. Appl Clin Inform 2015;6(03):506-520

9 Holden RJ, Karsh BT. The technology acceptance model: its past and its future in health care. J of Biomedical Inform 2009;43(01): 159-172

10 Yarbrough AK, Smith TB. Technology acceptance among physicians: a new take on TAM. Med Care Res Rev 2007;64(06): 650-672

11 Lee JD, Kirlik A, Danioff MJ, eds. The Oxford Handbook of Cognitive Engineering. New York, NY: Oxford University Press; 2013

12 Jex HR. Measuring mental workload: problems, progress, and promises. In: Hancock PA, Meshkati N, eds. Human Mental Workload. Amsterdam, The Netherlands: Elsevier; 1988:5-39

13 Noyes JM, Bruneau DP. A self-analysis of the NASA-TLX workload measure. Ergonomics 2007;50(04):514-519

14 Horsky J, Kaufman DR, Oppenheim MI, Patel VL. A framework for analyzing the cognitive complexity of computer-assisted clinical ordering. J Biomed Inform 2003;36(1,2):4-22

15 Barlow SE; Expert Committee. Expert committee recommendations regarding the prevention, assessment, and treatment of child and adolescent overweight and obesity: summary report. Pediatrics 2007;120(Suppl 4):S164-S192

16 Office of evidence based practice (EBP) - critically appraised topic: dairy intake and body composition. Available at: https:// www.childrensmercy.org/contentassets/752849f69a344ba4885 6b523af8c70c1/dairy.pdf. Accessed February 10, 2020

17 Shook RP, Halpin K, Carlson JA, et al. Adherence with multiple national healthy lifestyle recommendations in a large pediatric center electronic health record and reduced risk of obesity. Mayo Clin Proc 2018;93(09):1247-1255

18 Davis FD. Perceived usefulness, perceived ease of use, and user acceptance of information technology. MIS Quart Manage Inf Syst. 1989;13(03):319-339

19 Moroney WF, Biers DW, Eggemeier FT. Some measurement and methodological considerations in the application of subjective workload measurement techniques. Int J Aviat Psychol 1995;5 (01):87-106

20 Xiao YM, Wang ZM, Wang MZ, Lan YJ. [The appraisal of reliability and validity of subjective workload assessment technique and NASA-task load index] (Chinese). Zhonghua Lao Dong Wei Sheng Zhi Ye Bing Za Zhi 2005;23(03):178-181

21 Fusch PI, Ness LR. Are we there yet? Data saturation in qualitative research. Qual Rep 2015;20(09):1408-1416

22 Arndt BG, Beasley JW, Watkinson MD, et al. Tethered to the EHR: Primary care physician workload assessment using EHR event log data and time-motion observations. Ann Fam Med 2017;15(05): 419-426

23 Milat AJ, King L, Bauman AE, Redman S. The concept of scalability: increasing the scale and potential adoption of health promotion interventions into policy and practice. Health Promot Int 2013;28 (03):285-298

24 Milat AJ, Bauman A, Redman S. Narrative review of models and success factors for scaling up public health interventions. Implement Sci 2015;10:113

25 Sharifi M, Franz C, Horan CM, et al. Cost-effectiveness of a clinical childhood obesity intervention. Pediatrics 2017;140(05):e20162998

26 Rogers VW, Hart PH, Motyka E, Rines EN, Vine J, Deatrick DA. Impact of Let's Go! 5-2-1-0: a community-based, multisetting childhood obesity prevention program. J Pediatr Psychol 2013;38 (09):1010-1020

27 Venkatesh V, Davis FD. A theoretical extension of the technology acceptance model: four longitudinal field studies. Manage Sci 2000;46(02):186-204

28 Davis FD, Bagozzi RP, Warshaw PR. Extrinsic and intrinsic motivation to use computers in the workplace. J Appl Soc Psychol 1992;22(14):1111-1132 